\title{
Analysis of Atomic Displacement Parameters and Molecular Motion in Crystals
}

\author{
By W. Hummel, * A. Raselli and H.-B. Bürgi \\ Laboratory for Chemical and Mineralogical Crystallography, University of Bern, CH-3012 Bern, Switzerland
}

(Received 1 March 1990; accepted 30 May 1990)

\begin{abstract}
First experiences in using the new computer-graphics program PEANUT for visualization and real-time manipulation of atomic anisotropic displacement parameters (ADP's) are illustrated by several examples. Kinematic and mixed kinematic-dynamic models of molecular motion in crystals are derived from observed ADP's in an iterative procedure for bis $\left(\eta^{6}\right.$-benzene)chromium, hexacarbonylchromium and boron nitrilotriacetate. The examples show that ADP's of high precision contain detailed information not only on rigid-body molecular motion and lowfrequency intramolecular modes but also on intramolecular bending and stretching vibrations as well as on couplings between overall and intramolecular motion.
\end{abstract}

\section{Introduction}

Atomic anisotropic displacement parameters (ADP's) are determined routinely together with atomic coordinates during single-crystal structure analyses to account for the fact that atoms in a crystal are not stationary but move about their equilibrium positions with finite amplitudes. When atoms in different unit cells are distributed over more than one equilibrium position ADP's also absorb effects of disorder. ADP's, although abundant, have had a far smaller impact on the understanding of atomic and molecular motion and disorder than atomic coordinates have had on the understanding of the chemical bond. There are several reasons for this: (1) In general ADP's from routine structure analyses are no longer published, even though their information content has increased steadily as a consequence of improved methods of data collection and data analysis. (2) Methods and models to analyze ADP's in terms of motion and disorder are not very well developed. If ADP's are interpreted at all, it is usually within the framework of rigid-body motion of molecules. Sometimes the rigid-body model is slightly improved by taking into consideration a small number of internal degrees of freedom with

\footnotetext{
* Present address: Paul Scherrer Institute, CH-5232 Villigen PSI, Switzerland.
}

0108-7681/90/050683-10\$03.00 large amplitudes of motion (Johnson, 1970; Dunitz \& White, 1973; Dunitz, Schomaker \& Trueblood, 1988; Dunitz, Maverick \& Trueblood, 1988; Bürgi, 1989). Even for relatively sophisticated methods of analysis, agreement between model and observations is often poor. (3) The discrepancies ADP(obs) ADP(model) are usually available only as long lists of numbers and are difficult to analyze for trends because an appropriate (computer-) graphical tool has been lacking.

In order to improve this situation we have developed the computer-graphics program PEANUT (Hummel, Hauser \& Bürgi, 1990) which is designed primarily for the visualization and real-time manipulation of observed and calculated ADP's or of their differences. In this paper we describe first experiences in using this program. Several applications are illustrated by examples: (1) Comparison of ADP's from $\mathrm{X}$-ray and neutron diffraction; comparison of ADP's from different types of refinement, e.g. standard refinement and multipole refinement. (2) Comparison of observed ADP's with those calculated from models, e.g. simple rigid bodies, semi-rigid bodies undergoing some internal motion or flexible bodies with semi-empirical calculation of internal motions (by normal coordinate analysis).

\section{Mathematical background}

The probability density function $P(\mathbf{x})$ for finding an atom at a displacement $\mathbf{x}$ from equilibrium is usually assumed to be Gaussian

$$
P(\mathbf{x})=(2 \pi)^{-3 / 2}\left(\operatorname{det} \mathbf{U}^{-1}\right)^{1 / 2} \exp \left(-\mathbf{x}^{T} \mathbf{U}^{-1} \mathbf{x}\right) .
$$

$\mathbf{U}$ is the symmetric matrix of atomic mean-square displacements and may be represented graphically in terms of surfaces of constant probability,

$$
\mathbf{x}^{T} \mathbf{U}^{-1} \mathbf{x}=\text { constant. }
$$

Since mean-square displacements must be positive to be physically meaningful, the matrix $\mathbf{U}$ is positive definite. Thus (2) defines an ellipsoid with up to three different principal axes whose lengths correspond to anisotropic root-mean-square displacements; it is the basis for the well known ORTEP drawings (Johnson, 1976) of crystal and molecular structures.

(C) 1990 International Union of Crystallography 
Problems may arise if differences between meansquare displacement (MSD) tensors are to be represented, e.g. $\Delta \mathbf{U}=\mathbf{U}($ obs $)-\mathbf{U}($ model). If $\Delta \mathbf{U}$ is not positive definite, (2) no longer defines a closed surface and an alternative representation is needed. Following Nelmes (1969), we have chosen

$$
\begin{aligned}
\left\langle u^{2}(\mathbf{n})\right\rangle & =\mathbf{n}^{T} \mathbf{U n} \\
\left\langle\Delta u^{2}(\mathbf{n})\right\rangle & =\mathbf{n}^{T} \Delta \mathbf{U n} .
\end{aligned}
$$

The quantity $\mathbf{n}$ is a unit vector in an arbitrary direction; $\left\langle u^{2}(\mathbf{n})\right\rangle$ and $\left\langle\Delta u^{2}(\mathbf{n})\right\rangle$ are the MSD and difference MSD in that direction. For sufficiently different principal values of $\mathbf{U}$ the surface defined by $(3 a)$ looks remotely similar to a peanut and is therefore sometimes called peanut-shaped.

Root-mean-square displacements (RMSD's)

$$
\left\langle u^{2}(\mathbf{n})\right\rangle^{1 / 2}=\left(\mathbf{n}^{T} \mathbf{U n}\right)^{1 / 2}
$$

have the advantage of being comparable to ellipsoids of constant probability. Their magnitudes along the eigenvectors of $\mathbf{U}$ are identical to those of the ellipsoids along their principal axes if the constant in (2) equals one. By analogy to $(3 b)$ we also define

$$
\left\langle\Delta u^{2}(\mathbf{n})\right\rangle^{1 / 2}=\left(\mathbf{n}^{T} \Delta \mathbf{U n}\right)^{1 / 2} \text {. }
$$

The square root of the difference has been chosen rather than the difference of the square roots, because total mean-square displacements $\mathbf{U}$ may be considered as being composed of a large number of mean-square displacements, one for each of the vibrational degrees of freedom of the crystal (Willis $\&$ Pryor, 1975). The derivation of analytical formulae for these and some other types of representational surfaces of ADP's is discussed in detail by Nelmes (1969).

The surfaces defined by (3) and (4) are always closed. Throughout this paper, the positive parts of the MSD and RMSD surfaces are represented by their boundaries and by the visible parts of the MSD or RMSD curves in the three planes defined by pairs of principal axes of $\mathbf{U}$ or $\Delta \mathbf{U}$. The negative MSD's or imaginary RMSD's are shown as dotted surfaces.

\section{Comparison of different data sets}

\subsection{Different measurements}

Two low-temperature studies of hexacarbonylchromium, $\operatorname{Cr}(\mathrm{CO})_{6}$, have been reported: Fig. 1 $(a)$ shows the results of a high-order refinement $(\sin \theta / \lambda$ $\geq 0.76 \AA^{-1}$ ) based on an X-ray study at $74 \mathrm{~K}$ (Rees $\&$ Mitschler, 1976). Fig. 1(b) portrays the results of a neutron diffraction study at $78 \mathrm{~K}$ (Jost, Rees \& Yelon, 1975). It is difficult to spot obvious differences between the two measurements by visually comparing Figs. $1(a)$ and $1(b)$.
RMSD difference surfaces were therefore computed as follows: First the positional coordinates from the neutron data were rotated and translated to achieve an optimal overlay with the coordinates from X-ray data (algorithm after McLachlan, 1979). After this procedure the maximum distance between pairs of equivalent atoms is $0.005 \AA$, the average distance is $0.002 \AA$. Then, the ADP's U(neutron) were transformed accordingly and the differences $U(X-$ ray $)-$ U(neutron) calculated. Results are shown in Fig. $1(c)$. For better visibility, RMSD differences are scaled times $12 \cdot 5$. For $\mathrm{Cr}$ the differences between neutron and X-ray data are small. For $\mathrm{C}$ and $\mathrm{O}$ the ADP's from neutron data are generally larger than those from X-ray data; the differences are far from being isotropic. Most but not all of the large
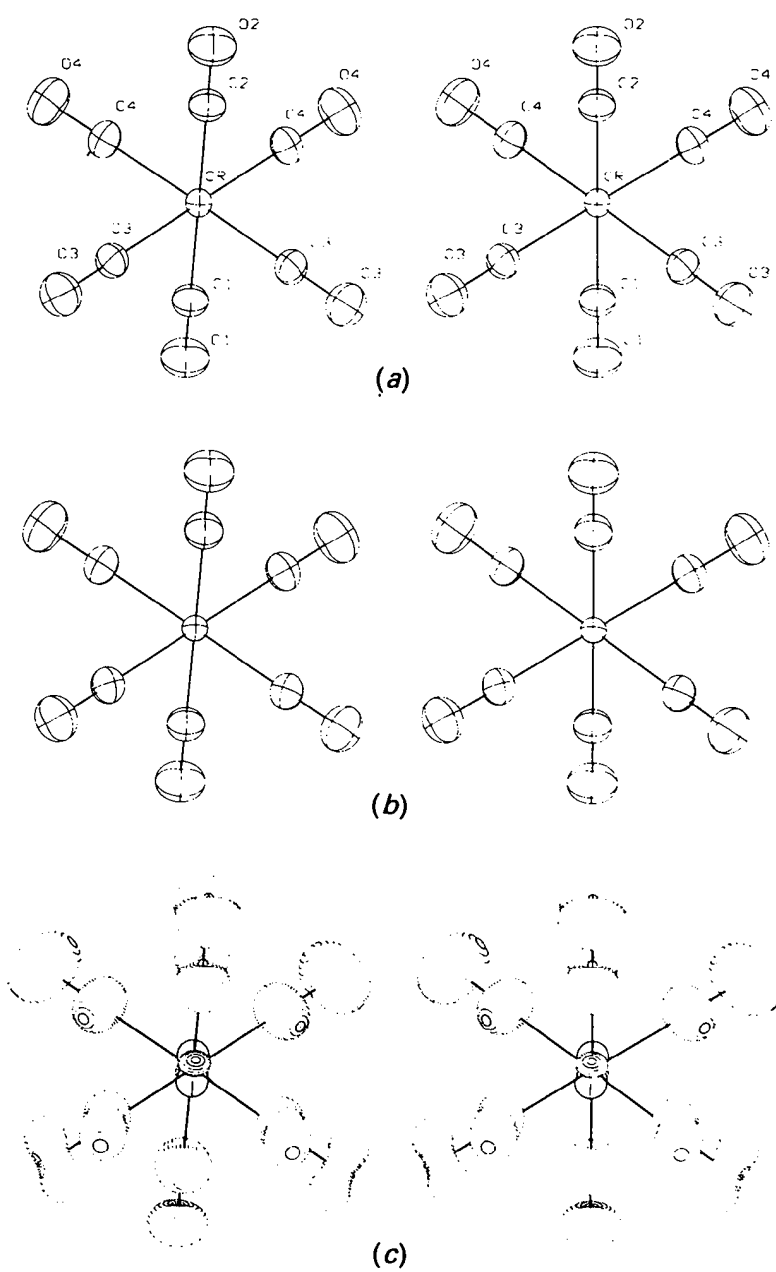

Fig. 1. (a) RMSD surfaces of hexacarbonylchromium from an $\mathrm{X}$-ray study at $74 \mathrm{~K}$ [U(X-ray), scale: $2 \cdot 5]$. (b) RMSD surfaces of hexacarbonylchromium from a neutron study at $78 \mathrm{~K}$ [U(neutron), scale: 2.5]. (c) Difference RMSD surfaces [U(X-ray) - U(neutron), scale: 12.5]. Outlines: positive; dots: imaginary. 
differences are found in a direction that is approximately parallel to the crystallographic $a$ axis (perpendicular to the plane of projection in Fig. 1) and might be due to unresolved extinction problems in the neutron data set as indicated by Rees $\&$ Mitschler (1976).

\subsection{Different refinements}

The new graphics tool may also be used to compare different refinements of the same experimental data. As an example, two refinements of boron nitrilotriacetate, $\mathrm{C}_{6} \mathrm{H}_{6} \mathrm{BNO}_{6}$, are discussed here (Moeckli, Schwarzenbach, Bürgi, Hauser \& Delley, 1988). Fig. 2(a) shows RMSD surfaces from a standard spherical-atom anisotropic refinement (std), whereas Fig. 2(b) displays results from a refinement with aspherical rigid atoms represented by $\kappa$ mono-

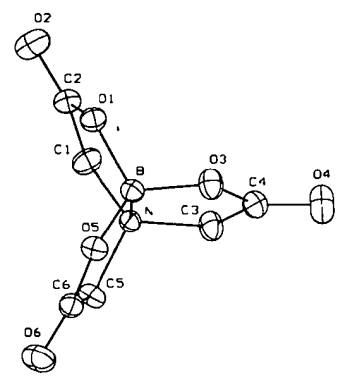

(a)
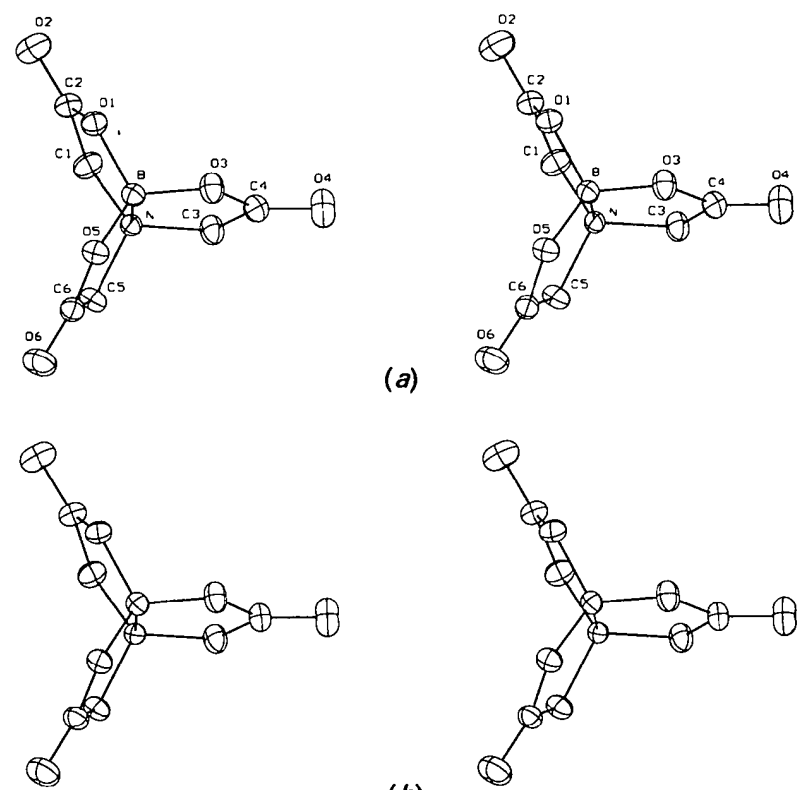

(b)
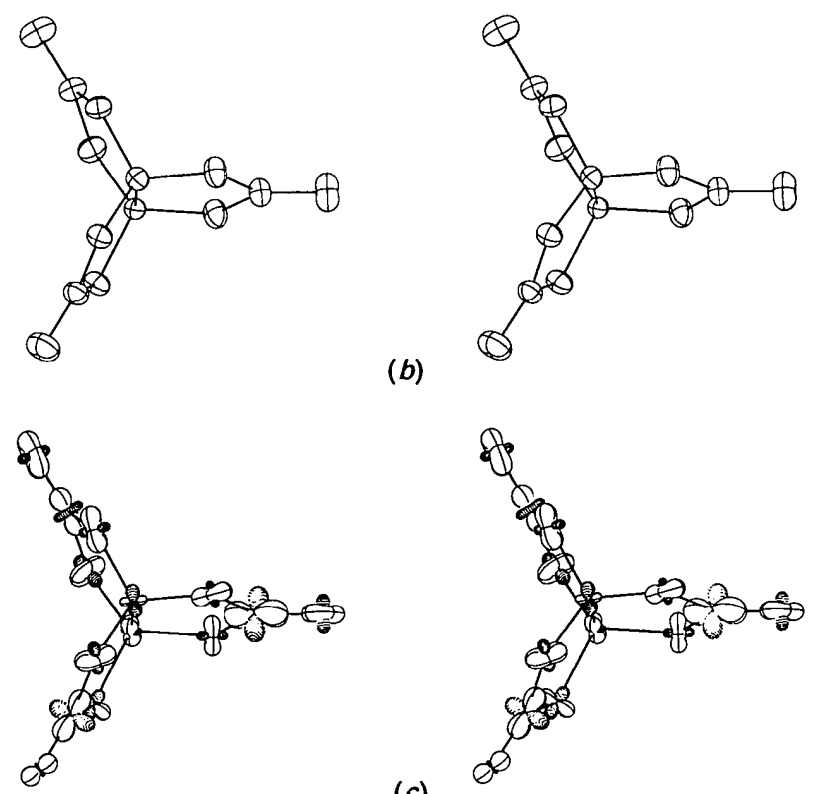

(c)

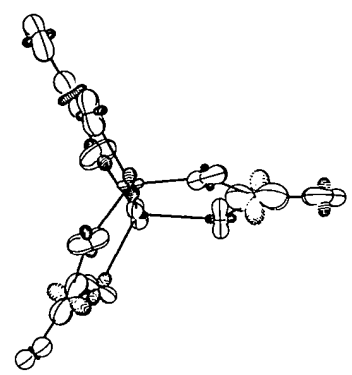

Fig. 2. (a) RMSD surfaces of boron nitrilotriacetate from a standard spherical-atom refinement [U(std), scale: $2 \cdot 5]$. (b) RMSD surfaces of boron nitrilotriacetate from a multipole refinement [U(kap), scale: 2.5]. (c) Difference RMSD surfaces [U(std) - U(kap), scale: 12.5]. Outlines: positive; dots: imaginary. poles, a multipole expansion and standard ADP's (kap). Careful scrutiny of the two pictures is necessary to identify differences between std and kap. Systematic trends are immediately obvious, however, from a picture of differences.

Again, the best overlay of the two data sets was computed (maximum distance between pairs of equivalent atoms is $0.005 \AA$, average distance $0.003 \AA)$, the differences $\mathbf{U}(\mathrm{std})-\mathbf{U}(\mathrm{kap})$ were calculated and are shown in Fig. 2(c) (RMSD surfaces scaled times 12.5). Although the molecule has no crystallographic symmetry, the difference RMSD surfaces (Fig. $2 c$ ) reflect the approximate molecular symmetry $3 \mathrm{~m}$. Thus, the picture is far from showing just random 'noise' of the two refinements. Rather, the differences are systematic and can be interpreted in a chemically significant way. The atoms $\mathrm{C} 2, \mathrm{C} 4$, $\mathrm{C} 6$ and $\mathrm{O} 2, \mathrm{O} 4, \mathrm{O} 6$ all have positive residuals in the direction of the $\mathrm{C}=\mathrm{O}$ bonds. This means that in the spherical-atom (std) refinement some bonding electron density of the $\mathrm{C}=\mathrm{O}$ bond is absorbed in the ADP's of C and O. A similar effect is seen for O1, $\mathrm{O} 3, \mathrm{O} 5$ and $\mathrm{N}$; the residuals parallel to the $\mathrm{B}-\mathrm{O}$ and $\mathrm{B}-\mathrm{N}$ bonds are all positive. The situation is different for $\mathrm{C} 1, \mathrm{C} 3$ and $\mathrm{C} 5$. Here, positive residuals are found perpendicular to the $\mathrm{C}-\mathrm{C}-\mathrm{N}$ planes. The C's all carry two $\mathrm{H}$ atoms (not shown in Figs. $2 a-c$ ) and in the std refinement the ADP's of $\mathrm{Cl}, \mathrm{C} 3$ and $\mathrm{C} 5$ seem to absorb some electron density of the $\mathrm{C}-\mathrm{H}$ bonds.

Thus, the ADP's of the spherical-atom refinement are more or less 'contaminated' by bonding electron density. In order to produce ADP's that are largely free from this contamination, high-order data or, preferably, multipole refinements are needed (see also: Dunitz, Maverick \& Trueblood, 1988).

\section{Kinematic and mixed kinematic-dynamic models of ADP's}

The terms 'kinematic' and 'dynamic' models are used here with the following meanings. A kinematic model attempts to give a description of molecular motion without considering the influence of the atomic masses and of the inter- and intramolecular forces explicitly. The model is usually defined in terms of three translational and three rotational motions (and their mean-square amplitudes) pertaining to the molecule as a whole (rigid-body model) and, sometimes, in terms of a limited number of additional motions pertaining to rigid segments of the molecule (segmented rigid body). The number of degrees of freedom is smaller, and often very much smaller, than the allowed maximum of three times the number of atoms in the molecule $(3 N)$. In contrast, a dynamical model takes explicit account of the interatomic forces and the atomic masses. In the 
present context dynamical models usually take the form of a normal coordinate model formulated in terms of a force-constant matrix and a reduced-mass matrix. Applied to a molecule in its averaged environment (molecular mean field) the model in its most general form has to account for $3 N$ degrees of motional freedom.

The simplest kinematic model for interpreting ADP's in terms of molecular motion is based on the assumption that a molecule may be considered as a rigid body in the crystal (Cruickshank, 1956; Schomaker \& Trueblood, 1968). The overall motion of the rigid body is described by a translation tensor $\mathbf{T}$, a libration tensor $\mathbf{L}$ and a coupling tensor $\mathbf{S}$. The observed ADP's (obs) are used in a straightforward linear least-squares procedure to calculate the best (in the least-squares sense) TLS parameters of the rigid body. If the assumption of a rigid body is valid, the model should reproduce the observed ADP's within experimental error, i.e.

$$
\mathrm{U}(\mathrm{obs}) \simeq \mathrm{U}(T L S) \text {. }
$$

The residuals $\mathbf{U}(\mathrm{obs})-\mathbf{U}(T L S)$ will be on the order of $\sigma[\mathbf{U}(\mathrm{obs})]$ and distributed randomly. If the residuals are large and/or show systematic features, the model is not adequate and should be improved.

A quick way to test how closely a molecule resembles a rigid body is to calculate difference MSD's along internuclear unit vectors $\mathbf{n}(i, j)$

$$
\left\langle\Delta u^{2}[\mathbf{n}(i, j)]\right\rangle=\mathbf{n}^{T}(i, j)[\mathbf{U}(\text { atom } i)-\mathbf{U}(\operatorname{atom} j)] \mathbf{n}(i, j) .(6)
$$

A necessary (although not sufficient) condition for a rigid body is that all $\left\langle\Delta u^{2}\right\rangle$ 's between atoms of the rigid body equal zero (Hirshfeld, 1976; Rosenfield, Trueblood \& Dunitz, 1978). An analogous condition applies to rigid groups within a molecule which as a whole may not be rigid (for a survey see: Dunitz, Maverick \& Trueblood, 1988).

A better description of molecular motion has to consider not only rigid-body motion but also the effect of internal molecular vibrations on the ADP's. The contributions to $\mathbf{U}(\mathrm{obs})$ from internal modes may be estimated from normal coordinate analysis (Wilson, Decius \& Cross, 1955; Cyvin, 1968; Willis \& Pryor, 1975; Decius \& Hexter, 1977).

The actual model building is now a two-step procedure. First, the MSD's pertaining to internal vibrations (iv) are subtracted from the observed ADP's. Second, the residuals, $\mathbf{U}($ obs $)-\mathbf{U}($ iv) are subjected to TLS analysis as described above. This mixed kinematic-dynamic model assumes

$$
\mathbf{U}(\text { obs }) \simeq \mathbf{U}(T L S)+\mathbf{U}(\mathrm{iv}) .
$$

Normal coordinate analyses are not always available. Some of the large-amplitude internal motions may then be modelled in a purely kinematical way by extending the TLS analysis (Johnson, 1970; Dunitz
\& White, 1973; Dunitz, Schomaker \& Trueblood, 1988; Dunitz, Maverick \& Trueblood, 1988; Bürgi, 1989). In such models, the overall translational and rotational motion is combined with large-amplitude internal rotation or translation. One or more rigid groups ( $\mathrm{rg}$ ), attached to a rigid frame are assumed to rotate about or translate along a specific direction. All kinematic parameters are determined by a leastsquares procedure. If the model is adequate, one should find

$$
\mathbf{U}(\mathrm{obs}) \simeq \mathbf{U}(T L S)+\sum \mathbf{U}(\mathrm{rg}) .
$$

In some cases it may be necessary to extend the model even further by combining both methods described above. If, for example, a normal coordinate analysis does not include some low-frequency mode, the latter may be modelled kinematically as an internal motion of a rigid group:

$$
\mathbf{U}(\text { obs }) \simeq \mathbf{U}(T L S)+\mathbf{U}(\mathrm{iv})+\sum \mathbf{U}(\mathrm{rg})
$$

Several examples are discussed in detail below. A modified version of the program ASYM20 (Hedberg, Iijima \& Hedberg, 1979) was used for normal coordinate analyses, and the programs ORSBA (Johnson, 1970; example 1 below) and THMA11 (Schomaker \& Trueblood, 1984; Dunitz, Schomaker \& Trueblood, 1988; examples 2 and 3 below) were used for segmented rigid-body analyses. The quantity GOF is used to compare the goodness-of-fit of different models:

$$
\mathrm{GOF}=\left\{\sum_{m} w[\mathbf{U}(\mathrm{obs})-\mathbf{U}(\text { model })]^{2} /(m-n)\right\}^{1 / 2}
$$

where $w$ is a weight $\left\{w=1 / \sigma^{2}[\mathbf{U}(\mathrm{obs})]\right\}, m$ the total number of independent ADP's, and $n$ the number of independent parameters, thus $(m-n)$ is the number of degrees of freedom.

\subsection{Example 1: bis $\left(\eta^{6}\right.$-benzene $)$ chromium}

$\operatorname{Bis}\left(\eta^{6}\right.$-benzene)chromium has been studied at $100 \mathrm{~K}$ and refined with all data $\left(\sin \theta / \lambda<0.86 \AA^{-1}\right)$ to $w R=0.027$ (Keulen, 1969; Keulen \& Jellinek, 1966). The molecule shows crystallographic site symmetry $\overline{3}$ with only two independent $\mathrm{C}$ atoms (Fig. $3 a$ ).

Rigid-body parameters were determined from observed ADP's and the difference surfaces U(obs) $-\mathbf{U}(T L S)$ are given in Fig. $3(b)$. The residuals of both independent $\mathrm{C}$ atoms are significant and their shapes and orientations very similar. The principal axes of the difference RMSD surfaces suggest a kind of corkscrew motion of the benzene ring relative to the chromium. More accurately, this motion may be described as a translational oscillation of the benzene ring in the direction of the threefold axis of the molecule coupled to a rotational oscillation about this axis. Note that Fig. $3(b)$ is more informative than the usual rigid-body test. Table 1 shows large 


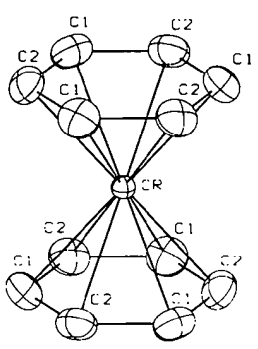

(a)
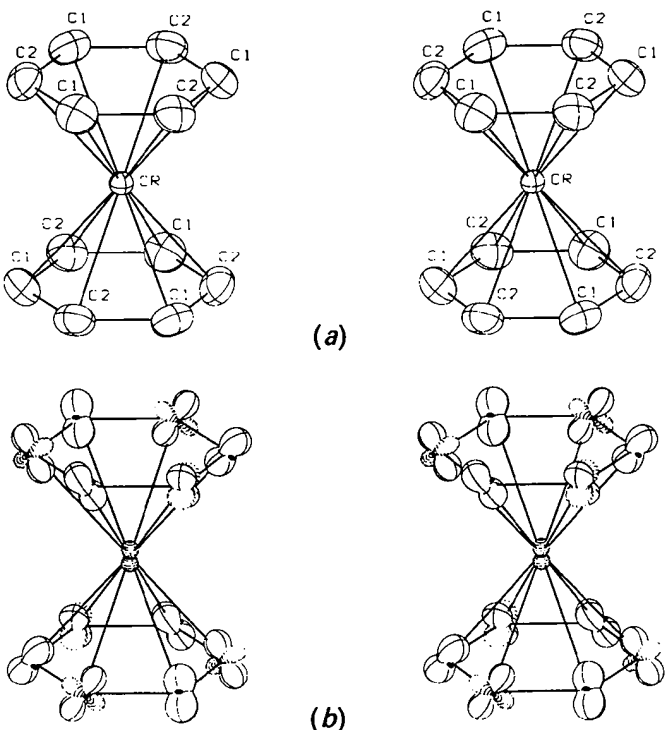

(b)
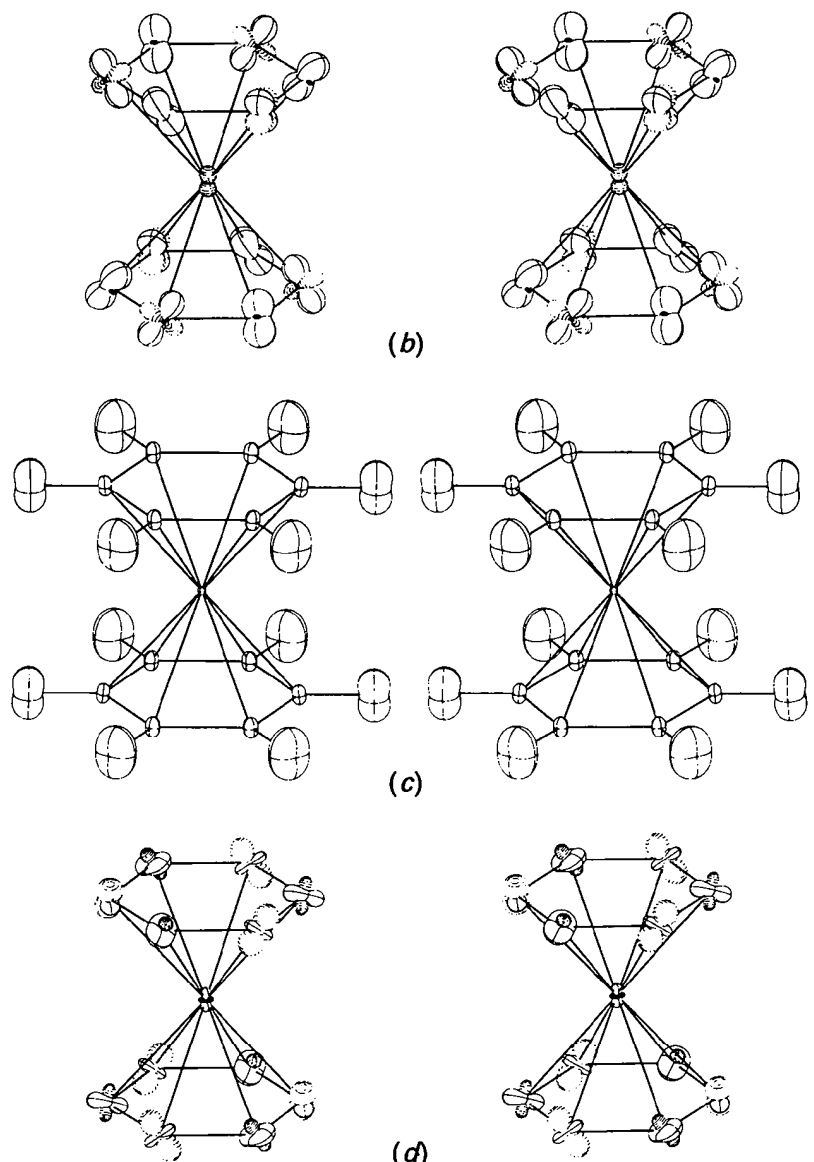

(d)
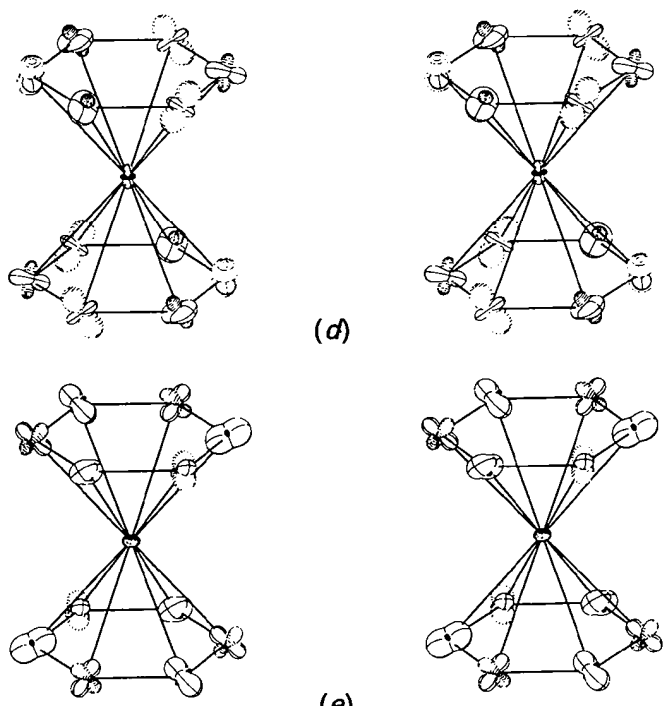

(e)

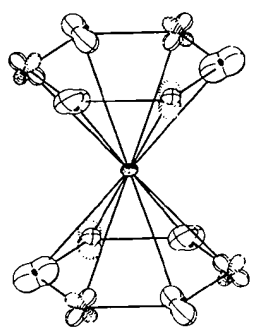

Fig. 3. (a) RMSD surfaces of bis $\left(\eta^{6}\right.$-benzene)chromium from an X-ray study at $100 \mathrm{~K}$ [U(obs), scale: $2 \cdot 5]$. (b) Difference RMSD surfaces [U(obs) - U(model I), scale: 10.0, Table 2]. (c) RMSD surfaces of $\operatorname{bis}\left(\eta^{6}\right.$-benzene)chromium (including $\mathrm{H}$ atoms) calculated from a normal coordinate analysis (scale: $2 \cdot 5) .(d)$ Difference RMSD surfaces [U(obs) - U(model II), scale: 10.0, Table 2]. (e) Difference RMSD surfaces [U(obs) - U(model III), scale: $10 \cdot 0$, Table 2]. Outlines: positive; dots: imaginary.
Table 1. Matrix of $\left\langle\Delta u^{2}\right\rangle$ 's $\left(10^{-4} \AA^{2}\right)$ for bis $\left(\eta^{6}\right.$-benzene $)$ chromium at $100 \mathrm{~K}$

Each $\left\langle\Delta u^{2}\right\rangle$ value is the MSD amplitude for the atom at the head of column minus the MSD amplitude for the atom at the left end of the row, each MSD amplitude being measured along the vector between the atoms. Values in parentheses are differences calculated from normal coordinate analysis. Average e.s.d.'s of U(obs) are $0.0001 \AA^{2}$ for $\mathrm{Cr}$ and $0.0005 \AA^{2}$ for C. The numbering scheme of the atoms is given in Fig. 3(a).

$\begin{array}{lccccccc} & \mathrm{C} 2(5) & \mathrm{Cl}(5) & \mathrm{Cl}(4) & \mathrm{C} 2(2) & \mathrm{Cl}(2) & \mathrm{C} 2(1) & \mathrm{Cl}(1) \\ \mathrm{Cr} & & & & & & 19 & 21 \\ & & & & & & (24) & (24) \\ \mathrm{Cl}(1) & -10 & 7 & 0 & -4 & 0 & -8 & \\ & (0) & (0) & (0) & (0) & (0) & (0) & \\ \mathrm{C} 2(1) & 15 & -7 & 4 & -1 & 5 & & \\ & (0) & (0) & (0) & (0) & (0) & & \end{array}$

$\left\langle\Delta u^{2}\right\rangle$ 's along the $\mathrm{C}-\mathrm{Cr}$ bonds and along $\mathrm{C} \cdots \mathrm{C}$ directions parallel to the $\overline{3}$ axis, but the inclination obvious from Fig. 3(b) would be difficult to extract from Table 1.

In order to improve the estimate of the rigid-body parameters, mean-square displacements were calculated from a normal coordinate analysis including all bond-stretching and angle-bending coordinates of the molecule and using vibrational frequencies observed in the solid state (Cyvin, Brunvoll \& Schäfer, 1971). The RMSD surfaces of $\operatorname{bis}\left(\eta^{6}-\right.$ benzene)chromium (including $\mathrm{H}$ atoms) calculated with site symmetry $6 / \mathrm{mmm}\left(D_{6 h}\right)$ are shown in Fig. $3(c)$. The largest vibrational amplitudes of $\mathrm{C}$ (and $\mathrm{H}$ ) are perpendicular to the benzene ring plane and larger than those of $\mathrm{Cr}$. Calculated values of $\left\langle\Delta u^{2}(\mathrm{C}, \mathrm{Cr})\right\rangle$ are equal to observed differences to within one standard deviation (Table 1). This is taken to indicate that the observed differences represent real information rather than systematic errors in the diffraction data absorbed into the refined ADP's.

The contribution to the ADP's due to the internal modes, U(iv), were subtracted from the observed ADP's, U(obs), and a rigid-body fit was performed on $\mathbf{U}($ obs $)-\mathbf{U}(\mathrm{iv})$. Fig. $3(d)$ shows the residuals $\mathbf{U}($ obs $)-\mathbf{U}(\mathrm{iv})-\mathbf{U}(T L S)$. Comparison with Fig. $3(b)$ indicates a better fit, the difference RMSD surfaces being distinctly smaller. And indeed, the goodness of fit (Table 2) drops from 2.5 (model I) to 1.8 (model II). Another effect seen from Table 2 is the general decrease of the librational and translational motion of the rigid body, which means that the $\mathbf{T}$ and $\mathbf{L}$ tensors of model I contain contributions from internal vibrations of the molecule.

Although model II, internal vibrations plus overall external motion, is a better approximation of the molecular motion than model I, the goodness of fit is not equal to its ideal value of 1.0 and thus some of the remaining features in Fig. 3(d) may be significant. The difference RMSD on C still shows a systematic inclination to the $\overline{3}$ axis. This results from the neglect in the normal coordinate analysis of coupling 
Table 2. Summary of (segmented) rigid-body-motion parameters for bis $\left(\eta^{6}\right.$-benzene $)$ chromium at $100 \mathrm{~K}$ (principal $R M S D$ of $\mathbf{L}$ in ${ }^{\circ}$ and $\mathbf{T}$ in $\AA$ )

$\begin{array}{lllll}\text { Model } & & I^{a} & \text { II }^{b} & \text { III }^{c} \\ L 1 & \| \overline{3} & 3 \cdot 10 & 3.01 & 3.21 \\ L 2=L 3 & \perp \overline{3} & 2.51 & 2 \cdot 23 & 2.39 \\ \text { E.s.d. } & & 0.08 & 0.06 & 0.05 \\ T 1 & \| \overline{3} & 0.0710 & 0.0610 & 0.0671 \\ T 2=T 3 & \perp \overline{3} & 0.0697 & 0.0659 & 0.0704 \\ \text { E.s.d. } & & 0.0011 & 0.0009 & 0.0007 \\ \left\langle\tau^{2}\right\rangle^{1 / 2} & \| \overline{3} & & & 0.0429(27) \\ \text { GOF } & & & & 1.44\end{array}$

Notes: (a) Model I: rigid-body fit on U(obs). (b) Model II: rigid-body fit on $\mathbf{U}$ (obs) - U(iv). (c) Model III: rigid-body motion combined with internal translation $\tau(\AA)$ of $\mathrm{C}_{6} \mathrm{H}_{6}$ along $\overline{3}$; allowance is made for coupling between rotation about $\overline{3}$ and internal translation $\left[\left\langle\tau l_{1}\right\rangle=0.036(8) \AA^{\circ}\right.$, for definition of symbols see Bürgi (1989)].

between the $\mathrm{Cr}$-benzene stretching motion and the rotation of the rings. As an alternative, a model combining overall rigid-body motion with internal translation of $\mathrm{C}_{6} \mathrm{H}_{6}$ in the direction of the $\overline{3}$ axis was analyzed (model III). Inclusion of a coupling term between internal translation and overall rotation reduced the goodness of fit to 1.4 . The positive residuals of $\mathrm{C}$ in Fig. 3(e) are comparable in magnitude to those in Fig. 3(d), but their orientation is different. They are only slightly inclined to the ring plane and pointing towards the molecular symmetry axis. This may reflect $\mathrm{C}-\mathrm{C}-\mathrm{C}$ angle bending not included in model III or a systematic error in the U(obs) owing to an aspherical electron density distribution in the direction of the $\mathrm{C}-\mathrm{H}$ bonds (see $\$ 3.2$, above) or both.

Comparison of the simple model I with the kinematic segmented rigid-body model III suggests a dynamical interpretation of the ADP's in which some of the $3 N-6$ internal vibrations of $\mathrm{Cr}\left(\mathrm{C}_{6} \mathrm{H}_{6}\right)_{2}$ are coupled to some of the six overall translations and rotations in the mean field of the surrounding crystal. Given the site symmetry $\overline{3}$, there are, among others, $\mathrm{Cr}$-benzene stretching motions belonging to the $A_{g}$ and $A_{u}$ irreducible representations, an internal rotation of one ring against the other $\left(A_{u}\right)$ and an overall rotation about $\overline{3}\left(A_{g}\right)$. The difference in the orientation of the residuals in models I and III (Figs. $3 b$ and $3 e$ ) is consistent with symmetry-allowed coupling between the $A_{g}$ and/or $A_{u}$ stretching vibrations and the internal and/or molecular rotations.

\subsection{Example 2: hexacarbonylchromium}

The second example deals with results of a highorder $\left(\sin \theta / \lambda \geq 0.76 \AA^{-1}\right)$ X-ray study of $\operatorname{Cr}(\mathrm{CO})_{6}$ at $74 \mathrm{~K}$ (Rees \& Mitschler, 1976) (Fig. la). The molecule has crystallographic site symmetry $m$. The following discussion closely follows that for $\mathrm{Cr}\left(\mathrm{C}_{6} \mathrm{H}_{6}\right)_{2}$ above.
The residuals $\mathbf{U}(\mathrm{obs})-\mathbf{U}(T L S)$ of a simple rigidbody fit to the experimental data are shown in Fig. 4(a) (model I). The difference RMSD surfaces reveal that $\mathrm{Cr}(\mathrm{CO})_{6}$ is far from being rigid. In particular,

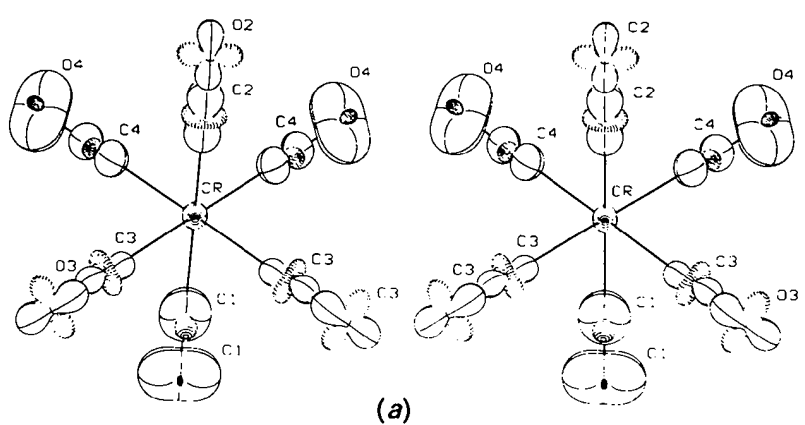

(a)

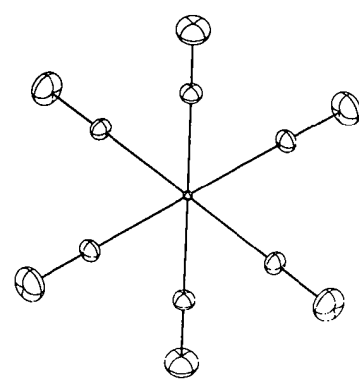

(b)
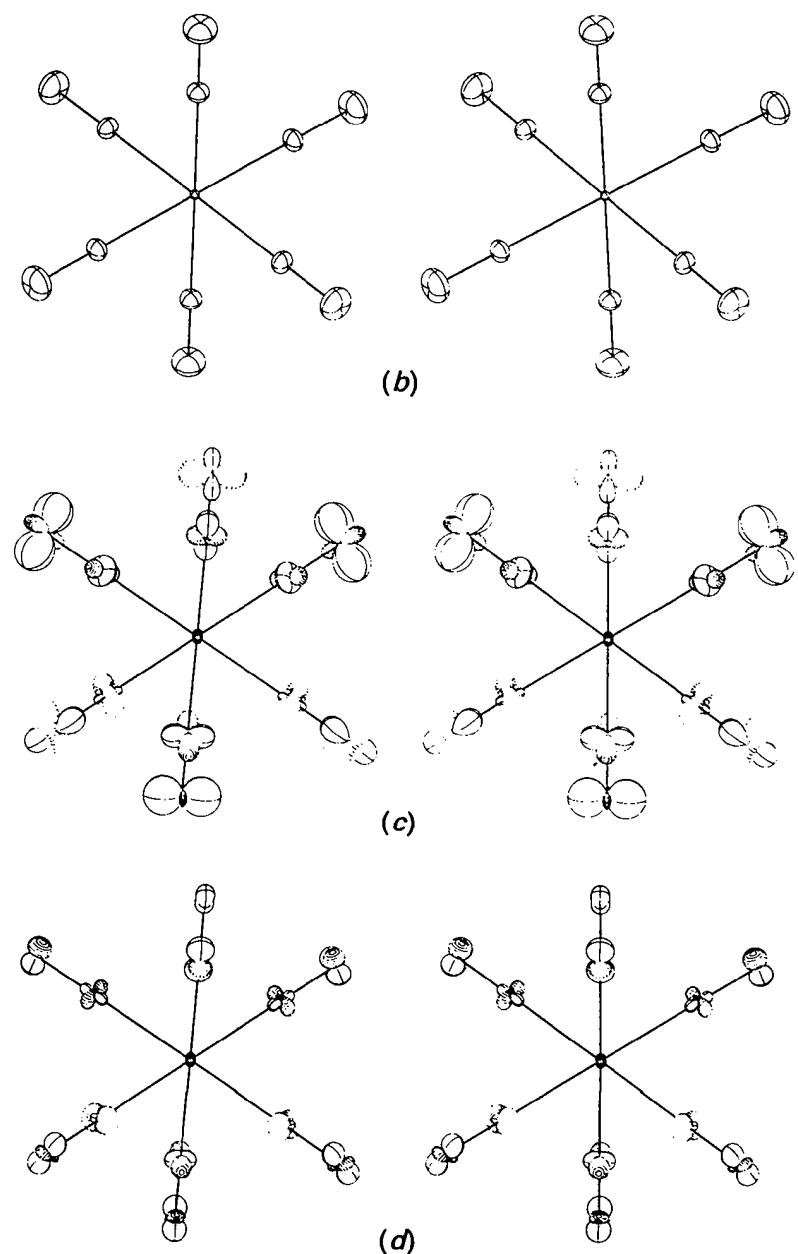

Fig. 4. (a) Difference RMSD surfaces of hexacarbonylchromium from an X-ray study at $74 \mathrm{~K}[U(\mathrm{obs})-\mathrm{U}$ (model I), scale: 12.5 , Table 4]. (b) RMSD surfaces of hexacarbonylchromium calculated from a normal coordinate analysis (scale: 2.5). (c) Difference RMSD surfaces [U(obs) - U(model II), scale: 12.5, Table 4]. (d) Difference RMSD surfaces [U(obs) - U(model III), scale: $12 \cdot 5$, Table 4]. Outlines: positive; dots: imaginary. 
Table 3. Matrix of $\left\langle\Delta u^{2}\right\rangle$ 's $\left(10^{-4} \AA^{2}\right)$ for hexacarbonylchromium at $74 \mathrm{~K}$

Each $\left\langle\Delta u^{2}\right\rangle$ value is the MSD amplitude for the atom at the head of column minus the MSD amplitude for the atom at the left end of the row, each MSD amplitude being measured along the vector between the atoms. Primed atoms are related by mirror symmetry to unprimed ones. Values in parentheses are differences calculated from normal coordinate analysis. Average e.s.d.'s of U(obs) are $0.0001,0.0006$ and $0.0005 \AA^{2}$ for $\mathrm{Cr}, \mathrm{O}$ and $\mathrm{C}$, respectively. For the numbering of atoms see Fig. $4(a)$.

$\begin{array}{lccccccccccc} & \mathrm{O}^{\prime} & \mathrm{O}^{\prime} & \mathrm{C} 4 & \mathrm{O} 4 & \mathrm{O} 3 & \mathrm{O} 2 & \mathrm{O} 1 & \mathrm{C} 4 & \mathrm{C} 3 & \mathrm{C} 2 & \mathrm{C} 1 \\ \mathrm{Cr} & & & & 15 & 14 & 19 & 14 & 19 & 16 & 27 & 17 \\ & & & & (14) & (14) & (14) & (14) & (15) & (15) & (15) & (15) \\ \mathrm{C} 1 & & & & 8 & -1 & 2 & -3 & 1 & -4 & 9 & \\ & & & & (6) & (6) & (-1) & (-1) & (0) & (0) & (0) & \\ \mathrm{C} 2 & & & & 7 & 13 & -8 & -12 & 0 & -3 & & \\ & & & & (6) & (6) & (-1) & (-1) & (0) & (0) & & \\ \mathrm{C} 3 & -1 & 10 & 3 & 23 & -1 & 12 & 11 & 9 & & & \\ & (-1) & (6) & (0) & (6) & (-1) & (6) & (6) & (0) & & & \\ \mathrm{C} 4 & 9 & -4 & & -4 & 2 & 2 & 10 & & & & \\ & (6) & (-1) & & (-1) & (6) & (6) & (6) & & & & \\ \mathrm{O} 1 & & & & -4 & -12 & 4 & & & & & \\ & & & & (0) & (0) & (0) & & & & & \\ \mathrm{O} 2 & & & & 4 & 4 & & & & & & \\ & & & & (0) & (0) & & & & & & \\ \mathrm{O} 3 & 1 & & & 18 & & & & & & & \\ & (0) & & & (0) & & & & & & & \end{array}$

the large positive residuals of $\mathrm{C}$ and $\mathrm{O}$ in the direction of the $\mathrm{C} \equiv \mathrm{O}$ bonds indicate significant contributions of $\mathrm{Cr}-\mathrm{CO}$ stretching modes to the observed ADP's in analogy to $\mathrm{Cr}\left(\mathrm{C}_{6} \mathrm{H}_{6}\right)_{2}$.

Results of a normal coordinate analysis for $\mathrm{Cr}(\mathrm{CO})_{6}$ with point symmetry $m \overline{3} m\left(O_{h}\right)$ and frequencies measured in $\mathrm{CCl}_{4}$ solution, in the gaseous and in the solid state (Jones, McDowell \& Goldblatt, 1969), were used to calculate MSD's due to the internal modes (Fig. 4b). In Table 3 the observed differences $\left\langle\Delta u^{2}\right\rangle$ between all pairs of atoms are compared to the values calculated from normal coordinate analysis. Mostly, there is fair agreement indicating that observed $\left\langle\Delta u^{2}\right\rangle$ 's are physically significant and not a result of systematic error in the diffraction measurements or of an inadequate refinement.

As in the previous example, the estimated internal ADP's, U(iv), were subtracted from U(obs) and a new rigid-body fit was carried out on $\mathbf{U}(\mathrm{obs})-\mathbf{U}$ (iv). The overall translational and librational motion decreases significantly (Table 4 , model II) and the goodness of fit drops from 2.7 (model I) to 1.9 (model II). Thus model II is a significantly better description of the experimental ADP's than the pure rigid-body model $\mathrm{I}$.

However, the goodness of fit is still larger than $1 \cdot 0$, indicating that some significant features in the experimental ADP's still remain unexplained by model II. A picture of difference surfaces $\mathbf{U}($ obs $)-$ $\mathbf{U}$ (model II) (Fig. 4c) shows large residuals of all $\mathrm{C}$ and $\mathrm{O}$ atoms perpendicular to their $\mathrm{C} \equiv \mathrm{O}$ bonds. They are of the same order of magnitude for all $\mathrm{O}$ atoms, positive for $\mathrm{O} 1, \mathrm{O} 4$ and $\mathrm{O}^{\prime}$ owing to the least-squares fit, negative for $\mathrm{O} 2, \mathrm{O} 3$ and $\mathrm{O}^{\prime}$. The $\mathrm{C}$ atoms show corresponding but smaller residuals.
Table 4. Summary of (segmented) rigid-body-motion parameters for hexacarbonylchromium at $74 \mathrm{~K}$ (principal RMSD of $\mathbf{L}$ in ${ }^{\circ}$ and $\mathbf{T}$ in $\AA$ )

$\begin{array}{lllll}\text { Model } & & \mathrm{I}^{a} & \mathrm{II}^{b} & \text { III }^{c} \\ L 1 & \simeq \| a & 2.27 & 1.41 & 0.80 \\ L 2 & \| b & 1.94 & 0.69 & 0.70 \\ L 3 & =\| c & 2.11 & 1.15 & 1.21 \\ \text { E.s.d. } & & 0.09 & 0.09 & 0.05 \\ T 1 & \simeq \| a & 0.0826 & 0.0775 & 0.0776 \\ T 2 & \| b & 0.0795 & 0.0737 & 0.0738 \\ T 3 & \simeq \| c & 0.0838 & 0.0781 & 0.0780 \\ \text { E.s.d. } & & 0.0015 & 0.0011 & 0.0006 \\ \left\langle\varphi^{2}\right\rangle^{1 / 2} & =\| a & & & 1.62(9) \\ \text { GOF } & & & & 0.90\end{array}$

Notes: (a) Model I: rigid-body fit of U(obs). (b) Model II: rigid-body fit of U(obs) - U(iv). (c) Model III: extension of model II by an additional rigid group comprising atoms $\mathrm{Cl}, \mathrm{O} 1, \mathrm{C} 4, \mathrm{O} 4, \mathrm{C}^{\prime}$ and $\mathrm{O}^{\prime}$ ' with a torsional vibration $\varphi\left({ }^{\circ}\right)$ about the axis $\mathrm{Cr}-$ center of $\mathrm{O} 1, \mathrm{O} 4, \mathrm{O}^{\prime}$. No significant coupling terms were found between internal rotation and the rigid-body motions, and thus coupling is neglected in model III.

Thus, model II was extended by including an extra torsional motion of $\mathrm{O} 1, \mathrm{O} 4, \mathrm{O}^{\prime}, \mathrm{C} 1, \mathrm{C} 4$ and $\mathrm{C}^{\prime}{ }^{\prime}$ about the approximate molecular threefold axis defined by $\mathrm{Cr}$ and the center of $\mathrm{O} 1, \mathrm{O} 4 \mathrm{O}^{\prime}$. The amplitude $\left\langle\varphi^{2}\right\rangle^{1 / 2}$ of this torsional vibration is larger than all molecular librations (Table 4, model III) and the goodness of fit drops to 0.9. Model III describes the experimental ADP's to within their e.s.d.'s and the residuals $\mathbf{U}$ (obs) - $\mathbf{U}$ (model III) do not contain any further information on molecular motion of $\mathrm{Cr}(\mathrm{CO})_{6}$ (Fig. $4 d$ ). The extra motion found for one half of the molecule but not for the other lends itself to a dynamical interpretation analogous to the one described for $\operatorname{Cr}\left(\mathrm{C}_{6} \mathrm{H}_{6}\right)_{2}$ : it may well be that the overall rotation of $\mathrm{Cr}(\mathrm{CO})_{6}$ is coupled with a specific $\mathrm{C}-\mathrm{Cr}-\mathrm{C}$ angle deformation coordinate, which is best described by rotational displacements in opposite directions of ligands $1,4,4^{\prime}$ and ligands $2,3,3^{\prime}$, respectively.

\subsection{Example 3: boron nitrilotriacetate}

In the last example, ADP's of relatively high precision from a multipole refinement of boron nitrilotriacetate (Moeckli, Schwarzenbach, Bürgi, Hauser \& Delley, 1988) are analyzed.

Model I, the simple rigid-body approach, yields very large residuals (Fig. $5 a$ ) and GOF $=5.0$ (Table 5). Significant positive differences $\mathbf{U}(\mathrm{kap})-\mathbf{U}(T L S)$ are found for the atoms $\mathrm{Cl}, \mathrm{C} 3, \mathrm{C} 5$ and $\mathrm{O} 1, \mathrm{O} 3, \mathrm{O} 5$ in directions perpendicular to the five-membered ring planes (Fig. 5a). A tentative interpretation is in terms of a low-frequency torsional vibration about the $\mathrm{B}-\mathrm{N}$ bond in which the motion of $\mathrm{Cl}, \mathrm{C} 3, \mathrm{C} 5$ is correlated and opposite in direction to that of $\mathrm{Ol}$, $\mathrm{O} 3$, O5. Such a mode is plausible in view of the cage structure where the conformations about the $\mathrm{B}-\mathrm{N}$, $\mathrm{N}-\mathrm{C}$ and $\mathrm{B}-\mathrm{O}$ bonds are all eclipsed. Torsional 
oscillation about $\mathrm{B}-\mathrm{N}$ deforms the conformations about the $\mathrm{B}-\mathrm{N}-$ and $\mathrm{N}-\mathrm{C}-$ bonds towards more favorable staggered arrangements, albeit at the expense of making the ester linkage $\mathrm{B}-\mathrm{O}(1,3,5)-\mathrm{C}(2,4,6)=\mathrm{O}(2,4,6)$ nonplanar. Model II includes a torsional oscillation about the $B-N$ bond. The fit obtained with this model is substantially better (Table 5), the goodness of fit dropping from 5.0 (model I) to 2.9 (model II). The root-meansquare amplitude of torsion is $1.8^{\circ}$, comparable in magnitude to the overall librational amplitude about the $\mathrm{B}-\mathrm{N}$ bond $\left[\left\langle\varphi^{2}(1)+2 \varphi(1) l^{\| \prime}\right\rangle^{1 / 2}\right.$ and $L(\mathrm{~B}-\mathrm{N})$ in Table $5^{*}$.

In the next step (model III), the systematic positive residuals of $\mathrm{O} 2, \mathrm{O} 4, \mathrm{O} 6$ and, as a consequence of the least-squares fit, the negative ones of $\mathrm{C} 2, \mathrm{C} 4, \mathrm{C} 6$ (Fig. $5 b$ ) may be interpreted as resulting from the motion of the carbonyl $\mathrm{O}$ atoms perpendicular to the planes of the ester groups. The out-of-plane amplitudes of $\mathrm{O} 2, \mathrm{O} 4$ and $\mathrm{O} 6$ are all of the same order of magnitude and reflect once again the approximate threefold molecular symmetry. The motion is modelled in terms of a single rotation of atoms $\mathrm{O} 2$, $\mathrm{O} 4$ and $\mathrm{O} 6$ about the $\mathrm{B}-\mathrm{N}$ bond. Its amplitude is $0.9^{\circ}\left[\left\langle\varphi^{2}(2)+2 \varphi(2) l^{\|}\right\rangle^{1 / 2}\right.$ in Table 5] which shows that the out-of-plane motion is significant. The absolute out-of-plane displacements of $\mathrm{O} 2, \mathrm{O} 4$ and $\mathrm{O} 6$ are of the same order of magnitude $(\simeq 0.05 \AA)$ as those of $\mathrm{O} 1, \mathrm{O} 3, \mathrm{O} 5$ and $\mathrm{Cl}, \mathrm{C} 3, \mathrm{C} 5$.

In model II above, the torsional oscillation of $\mathrm{Ol}$, $\mathrm{O} 3, \mathrm{O} 5$ and $\mathrm{C} 1, \mathrm{C} 3, \mathrm{C} 5$ about $\mathrm{B}-\mathrm{N}$ has been modelled with the same amplitude. As can be seen from Fig. $5(b)$ this is not quite adequate: the $\mathrm{O}$ atoms all have negative residuals perpendicular to the five-membered rings and the residuals of the $\mathrm{C}$ atoms are all positive in the same direction. Thus, the rigid group with internal rotation $\varphi(1)$ was split into two groups $\mathrm{C} 1, \mathrm{C} 3, \mathrm{C} 5$ with rotation $\varphi(1 a)$ and $\mathrm{O} 1, \mathrm{O} 3$, O5 with rotation $\varphi(1 b)$. The fit obtained for model IV (Table 5) is only slightly better than for model III, but, as expected, the torsional amplitude of the C atoms is significantly larger than the amplitude of the heavier $\mathrm{O}$ atoms.

Another significant feature concerns B, the lightest non- $\mathrm{H}$ atom in the molecule. It shows a large positive and almost isotropic difference RMSD surface (Figs. $5 a-c$ ). Chemical intuition suggests this to be a result of the light mass of $B$ and to the fact that bond-stretching and angle-bending force constants about $\mathrm{B}$ are lower than those about $\mathrm{C}, \mathrm{N}$ and $\mathrm{O}$. In

* A table of numerical values of the coupling terms (between internal rotation and overall rotation and overall translation) and their e.s.d.'s has been deposited with the British Library Document Supply Centre as Supplementary Publication No. SUP 53231 ( 3 pp.). Copies may be obtained through The Technical Editor, International Union of Crystallography, 5 Abbey Square, Chester CHI 2HU, England. order to test the influence of this motion on the TLS parameters, the B atom was excluded from the leastsquares fit (Table 5, model V). As expected, the overall translation T decreases; GOF drops to $2 \cdot 0$. The libration parameters show no significant changes.

The residuals of model $\mathrm{V}$ (Fig. $5 c$ ) are very small except for B; however, the difference RMSD surfaces of the cage atoms still reflect the approximate threefold molecular symmetry and GOF $\gg 1 \cdot 0$. This may be indicative of high-frequency angle-bending modes not accounted for in the present model.

\section{Discussion}

Early attempts to derive information about intramolecular motion from ADP's were also based on

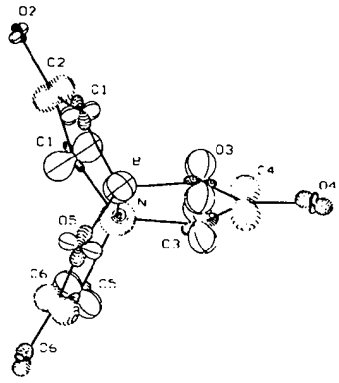

(a)
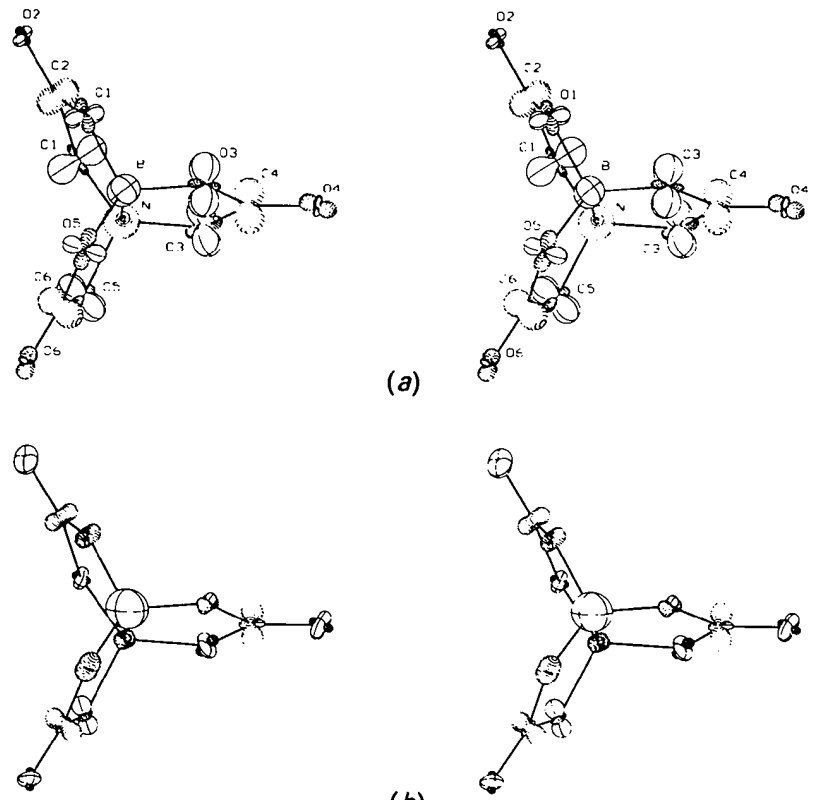

(b)
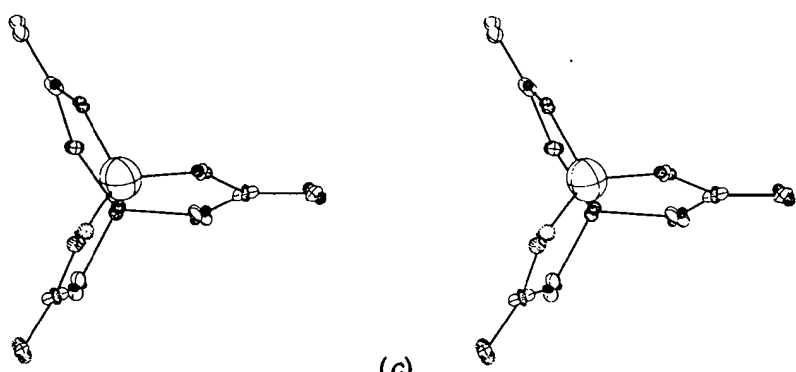

(c)

Fig. 5. (a) Difference RMSD surfaces of boron nitrilotriacetate [U(obs) - U(model I), multipole refinement, scale: $12 \cdot 5$, Table 5]. (b) Difference RMSD surfaces [U(obs) - U(model II), scale: 12.5, Table 5]. (c) Difference RMSD surfaces [U(obs) U(model V), scale: $12 \cdot 5$, Table 5]. Outlines: positive; dots: imaginary. 
Table 5. Summary of (segmented) rigid-body-motion parameters for boron nitrilotriacetate at $100 \mathrm{~K}$ (principal RMSD of $\mathrm{L}$ in ${ }^{\circ}$ and $\mathbf{T}$ in $\AA$ )

Average e.s.d.'s of $\mathrm{U}$ (obs) are $0.00012(\mathrm{O}), 0.00011(\mathrm{~N}), 0.00013$ (C) and $0.00014 \AA^{2}(\mathrm{~B})$.

\begin{tabular}{|c|c|c|c|c|c|}
\hline Model & $\mathrm{I}^{a}$ & $\mathrm{II}^{b}$ & III $^{b}$ & $\mathrm{IV}^{b}$ & $\mathbf{V}^{c}$ \\
\hline$L 1$ & 1.87 & 1.86 & 1.73 & 1.72 & 1.75 \\
\hline$L 2$ & 1.70 & 1.68 & 1.66 & 1.65 & 1.67 \\
\hline$L 3$ & 1.61 & 1.59 & 1.53 & 1.55 & 1.57 \\
\hline E.s.d. & 0.07 & 0.04 & 0.05 & 0.06 & 0.04 \\
\hline$T 1$ & 0.0780 & 0.0752 & 0.0759 & 0.0762 & 0.0751 \\
\hline$T 2$ & 0.0741 & 0.0723 & 0.0727 & 0.0730 & 0.0719 \\
\hline$T 3$ & 0.0713 & 0.0709 & 0.0717 & 0.0711 & 0.0704 \\
\hline E.s.d. & 0.0013 & 0.0009 & 0.0007 & 0.0008 & 0.0007 \\
\hline \multirow{4}{*}{\multicolumn{2}{|c|}{$\begin{array}{l}L(\mathrm{~B}-\mathrm{N}) \\
\left\langle\varphi^{2}(1)+2 \varphi(1) l^{1}\right\rangle^{1 / 2 d} \\
\left\langle\varphi^{2}(1 a)+2 \varphi(1 a) l^{1 / 2 d}\right\rangle^{1 / 2 d} \\
\left\langle\varphi^{2}(1 b)+2 \varphi(1 b) l^{1 / 2 d}\right. \\
\left\langle\varphi^{2}(2)+2 \varphi(2) l^{\prime}\right\rangle^{1 / 2 d}\end{array}$}} & $1.83(4)$ & $1.63(5)$ & $1.63(6)$ & $1.66(4)$ \\
\hline & & $1.78(9)$ & $1.91(7)$ & & \\
\hline & & & & $\begin{array}{l}2.03(8) \\
1.77\end{array}$ & $2.03(6)$ \\
\hline & & & $0.88(8)$ & $0.88(8)$ & $0.84(6)$ \\
\hline GOF & 5.00 & 2.89 & 2.52 & 2.39 & $2 \cdot 00$ \\
\hline
\end{tabular}

Notes: (a) Model I: rigid-body fit on U(obs). (b) Models II-IV; extensions of model I by including one (II), two (III), or three (IV) additional torsional vibrations with amplitude $\varphi(n)$ about the $\mathrm{B}-\mathrm{N}$ bond. $\varphi(1)$ affects atoms $\mathrm{Cl}, \mathrm{C} 3, \mathrm{C} 5, \mathrm{O} 1, \mathrm{O} 3$ and $\mathrm{O} 5 . \varphi(1 a)$ affects $\mathrm{Cl}, \mathrm{C} 3, \mathrm{C} 5$ only, whereas $\varphi(1 b)$ affects $\mathrm{O} 1, \mathrm{O} 3, \mathrm{O}, \varphi(2)$ affects atoms $\mathrm{O} 2, \mathrm{O} 4$ and $06 . L(\mathrm{~B}-\mathrm{N})$ is the overall (rigid-body) libration about the $\mathrm{B}-\mathrm{N}$ bond. (c) Model V: same as model IV but B excluded from the least-squares fit. (d) Coupling terms between internal rotation and overall rotation $\left(\left\langle\varphi l_{1}^{\perp}\right\rangle,\left\langle\varphi l_{2}^{\perp}\right\rangle\right)$ and overall translation $\left(\left\langle\varphi t^{\downarrow}\right\rangle,\left\langle\varphi t_{1}^{1}\right\rangle,\left\langle\varphi t_{2}^{1}\right\rangle\right)$ were also refined (see deposition footnote). The contributions $\left\langle\varphi^{2}\right\rangle$ and $\left\langle\varphi l^{l}\right\rangle$ cannot be obtained separately [for definition of symbols see Dunitz, Schomaker \& Trueblood (1988) and Bürgi (1989)].

the observation that observed ADP's can sometimes not be explained in terms of rigid-body motion. Typical examples were: H/D with large MSD's perpendicular to $\mathrm{C}-\mathrm{H} / \mathrm{D}$ bonds in aromatic hydrocarbons (Johnson, 1970), $X$ atoms in tetrahedral $-X Y_{3}$ groups or planar $-X Y_{2}$ groups with large MSD's of $Y$ perpendicular and tangential to the local symmetry axis (Trueblood \& Dunitz, 1983), and $\mathrm{C}$ atoms in $\eta^{6}$-benzene or $\eta^{5}$-cyclopentadienyl transition-metal complexes (Maverick \& Dunitz, 1987). In these cases visual inspection of the $\mathbf{U}($ obs) was often sufficient to suggest a hypothesis on the nature of the intramolecular motion. With the new graphics program $P E A N U T$ it is possible to analyze difference surfaces $[\mathbf{U}(\mathrm{obs})-\mathbf{U}(T L S)]$ for internal motions which are not apparent from the numerical listings of the differences or from $\mathbf{U}$ (obs) themselves.

The examples discussed above suggest an iterative procedure to derive kinematic or mixed kinematicdynamic models of motion from observed ADP's: First a rigid-body model is fitted to the experimental U's. Then its deficiencies are visualized in terms of the differences between observations and calculations, an improved model is formulated and the whole procedure is repeated until no significant features are left in $\mathbf{U}(\mathrm{obs})-\mathbf{U}$ (model) or until the models need to be more flexible than those presently accessible through the programs THMA11 and
ORSBA. With both of them a limited range of kinematical models including only internal and overall translation and rotation may be analyzed. A future generation of models should probably be based on a normal coordinate approach in which stretching, bending, rotational and translational force constants are derived in the molecular meanfield approximation from a combination of vibrational frequencies and ADP's. The examples analyzed in this paper and many others studied in our laboratory indicate that difference RMSD surfaces may be as important for modelling ADP's in terms of intramolecular motion as difference electron density maps have been and continue to be for determining and refining the geometrical structure and the detailed electron density distribution of molecules in crystals.

The examples given in this paper illustrate a number of specific points:

(1) The patterns of differences $\mathbf{U}($ obs) $-\mathbf{U}$ (model) have been analyzed for molecules whose molecular symmetry is higher than their site symmetry. The symmetry of the differences is generally higher than the crystallographic site symmetry and may even be as high as the molecular symmetry. This indicates that there is physically meaningful information about intramolecular motion in ADP's.

(2) Apart from the features caused by lowfrequency internal modes, information may be gleaned on couplings between internal and overall molecular motion and probably also on couplings between internal degrees of freedom.

(3) U's of very high quality are obtained as a byproduct from accurate high-order diffraction experiments or, preferably, from careful difference electron density studies based on multipole refinements. Their e.s.d.'s may be as low as $\simeq 0.00005 \AA^{2}$ (Hirshfeld, 1984), i.e. about equal to or even smaller than the MSD's typical of bondstretching and angle-bending motions. This opens up the possibility of extracting information from ADP's on the stiffer modes as well.

There is no doubt that practical experience with improved models of motion will lead back to an old problem, namely the extent to which observed ADP's are still affected by random and systematic errors arising from absorption, extinction, scan truncation, anharmonic motion, etc. However, for the time being it seems that much can still be learned on atomic and molecular motion in crystals from harmonic ADP's obtained from state-of-the-art diffraction data.

We gratefully acknowledge financial help by the Schweizerischer Nationalfonds zur Förderung der wissenschaftlichen Forschung. We thank Professor F. Jellinek for a copy of E. Keulen's PhD Thesis. 


\section{References}

Bürgi, H.-B. (1989). Acta Cryst. B45, 383-390.

Cruickshank, D. W. J. (1956). Acta Cryst. 9, 754-756.

Cyvin, S. J. (1968). Molecular Vibrations and Mean-Square Amplitudes. Amsterdam: Elsevier.

Cyvin, S. J., Brunvoll, J. \& SChäfer, L. (1971). J. Chem. Phys. $54,1517-1522$.

Decius, J. C. \& Hexter, R. M. (1977). Molecular Vibrations in Crystals. New York: McGraw-Hill.

Dunitz, J. D., Maverick, E. F. \& Trueblood, K. N. (1988). Angew Chem. 100, 910-926.

Dunitz, J. D., Schomaker, V. \& Trueblood, K. N. (1988). J. Phys. Chem. 92, 856-867.

Dunirz, J. D. \& White, D. N. J. (1973). Acta Cryst. A29, 93-94.

Hedberg, L., Iljima, T. \& Hedberg, K. (1979). J. Chem. Phys. 70, 3224-3229.

HiRSHFEld, F. L. (1976). Acta Cryst. A32, 239-244.

HIRSHFELD, F. L. (1984). Acta Cryst. B40, 484-492.

Hummel, W., HaUSER, J. \& BÜRGI, H.-B. (1990). In preparation.

Johnson, C. K. (1970). Thermal Neutron Diffraction, edited by B. T. M. WILlis, pp. 132-160. Oxford Univ. Press.

JoHnson, C. K. (1976). ORTEPII. Report ORNL-5138. Oak Ridge National Laboratory, Tennessee, USA.

JoNes, L. H., MCDowell, R. S. \& GoldblatT, M. (1969). Inorg. Chem. 8, 2349-2363.
Jost, A., Rees, B. \& Yelon, W. B. (1975). Acta Cryst. B31, 2649-2658.

Keulen, E. (1969). PhD Thesis, Univ. of Groningen, The Netherlands.

Keulen, E. \& Jellinek, F. (1966). J. Organomet. Chem. 5, 490-492.

MCLachlan, A. D. (1979). J. Mol. Biol. 128, 49-79.

Maverick, E. F. \& Dunitz, J. D. (1987). Mol. Phys. 62, 451459.

Moeckli, P., Schwarzenbach, D., Bürgi, H.-B., Hauser, J. \& Delley, B. (1988). Acta Cryst. B44, 636-645.

Nelmes, R. J. (1969). Acta Cryst. A25, 523-526.

Rees, B. \& Mitschler, A. (1976). J. Am. Chem. Soc. 98, 79187924.

Rosenfield, R. E. JR, Trueblood, K. N. \& Dunitz, J. D. (1978). Acta Cryst. A34, 828-829.

SChOMAKeR, V. \& TRUEBloOd, K. N. (1968). Acta Cryst. B24, 63-76.

Schomaker, V. \& Trueblood, K. N. (1984). Acta Cryst. A40, C-339.

Trueblood, K. N. \& Dunitz, J. D. (1983). Acta Cryst. B39, 120-133.

Willis, B. T. M. \& PryoR, A. W. (1975). Thermal Vibrations in Crystallography. Cambridge Univ. Press.

Wilson, E. B. JR, Decius, J. C. \& Cross, P. C. (1955). Molecular Vibrations. New York: McGraw-Hill.

\section{SHORT COMMUNICATION}

Contributions intended for publication under this heading should be expressly so marked; they should not exceed about 1000 words; they should be forwarded in the usual way to the appropriate Co-editor; they will be published as speedily as possible.

Acta Cryst. (1990). B46, 692

Crystal structure and nonlinear optical properties of $\mathrm{KSnOPO}_{4}$ and their comparison with $\mathrm{KTiOPO}_{4}$. Erratum. By P. A. Thomas, A. M. GlaZER and B. E. WatTs, Clarendon Laboratory, Parks Road, Oxford OX1 3PU, England

(Received 30 August 1990)

\section{Abstract}

Some incorrect features in Fig. 1 of Thomas, Glazer \& Watts [Acta Cryst. (1990), B46, 333-343] are corrected. The sense of the $a$ axis is reversed, and the atom labels P(1) and $\mathrm{P}(2)$ are interchanged.

A corrected version of the illustration is shown in Fig. 1.

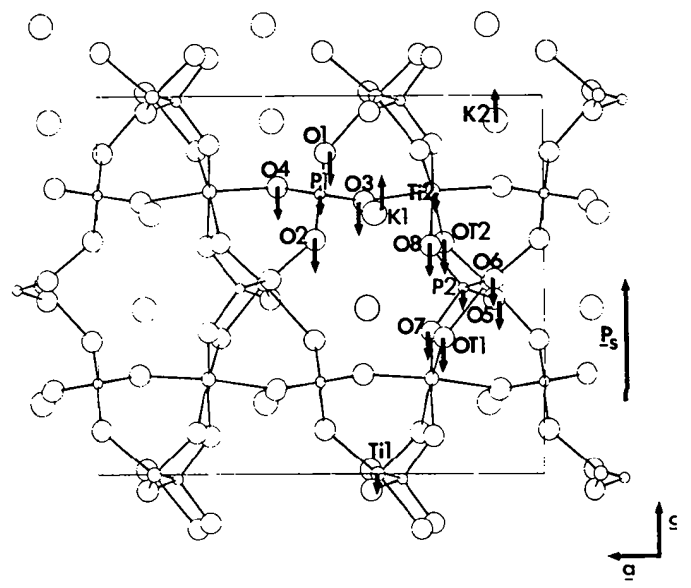

Fig. 1. A view of the KTP structure seen along [010] showing the directions for the calculated displacements of all atomic species in the transition from the prototype phase to the roomtemperature phase. The direction of the resultant spontaneous polarization, $\mathbf{P}_{s}$, is shown parallel with the chosen direction of $+\mathbf{c}$.

C 1990 International Union of Crystallography 